\title{
INTERACTIVE PROCEDURE FOR RAPID PERFORMANCE ESTIMATES OF MAGNET DESIGNS*
}

\author{
Carl L. Goodzeit", M. J. (Penny) Ball, M J B Plus Inc., DeSoto, TX, \\ Rainer B. Meinke, Advanced Magnet Lab Inc., Palm Bay, FL
}

\section{Abstract}

This paper illustrates the use of a simple interactive magnetic field calculator (IMC) program to estimate field strength and allowed harmonics for cosine theta dipole or quadrupole magnets. Niobium-tin coil designs from Saclay and the University of Twente are used as examples, and the IMC results are compared with the values from the magnet designers.

\section{MAGNETIC FIELD CALCULATOR}

One of the features included in a CD-ROM tutorial on superconducting accelerator magnets [1] is an interactive procedure for calculating magnetic field strength and allowed field harmonics in single layer cosine theta dipole or quadrupole magnets. This procedure uses a simplified geometry for the coil, which can contain a single wedge and an optional iron yoke. The yoke is defined by its inner radius and is assumed to be iron with infinite $\mu$.

However, this interactive magnetic field calculator (IMC) can be applied to more complicated magnet structures, consisting of multiple layers with several wedges, by use of superposition, i.e., decomposing the structure into several simple ones and adding the results. Using this technique, the results from the IMC are in good agreement with precise calculations for the central field, the peak field, and the $\mathrm{x}$ and $\mathrm{y}$ components of the Lorentz force. The allowed multipoles are in good agreement only when the conductor blocks and wedges are radially disposed (i.e. without any tilt). The details of using the interactive magnetic field calculator (IMC) have been previously described [2]. Input values define the coil geometry and the total current flow. Output includes the field strength and normalized multipole coefficients, a color-coded graph of the field strength in the conductor region, and the Lorentz forces on the coil.

The calculation procedure uses an ideal approximation of the coil geometry in which the conductor block and wedge edges are assumed to be radial. The input data consists of the inner and outer radius of the coil (R1 and R2), the start and stop angles of the coil (A1 and A2), the inner radius of the optional yoke (Ryoke), the start and stop angles of the optional wedge (A3 and A4), the average current density in the conductor area $(\mathrm{J})$, and the radius and angle at which field values are calculated (Rref and Aref). The data are entered into a graphical user interface which then displays the defined cross section.

\footnotetext{
*Work supported in part by U.S. Dept. of Energy SBIR grant \# DEFG03-94ER-81813

"Email: carl@mjb-plus.com
}

\section{SACLAY QUADRUPOLE EXAMPLE}

Saclay developed a protoype design for a quadrupole magnet with the LHC quadrupole dimensions but using a $\mathrm{Nb}_{3} \mathrm{Sn}$ conductor. This magnet coil has two layers with tilted current blocks [3]; the inner and outer layer coil cross sections are shown in Figure 1.

In order to use the IMC, this cross section is idealized into a radially disposed configuration; the angles for the idealized coil were selected to provide an average fit with the tilted coil blocks. The parameters for the LHC quadrupole magnet and those used for the idealized coils are summarized in Table 1 . The average current density for the idealized coils was obtained by dividing the product of the number of turns ( 10 for the inner and 14 for the outer coil) and the operating current $(11870 \mathrm{~A})$ by the area of the idealized current blocks.

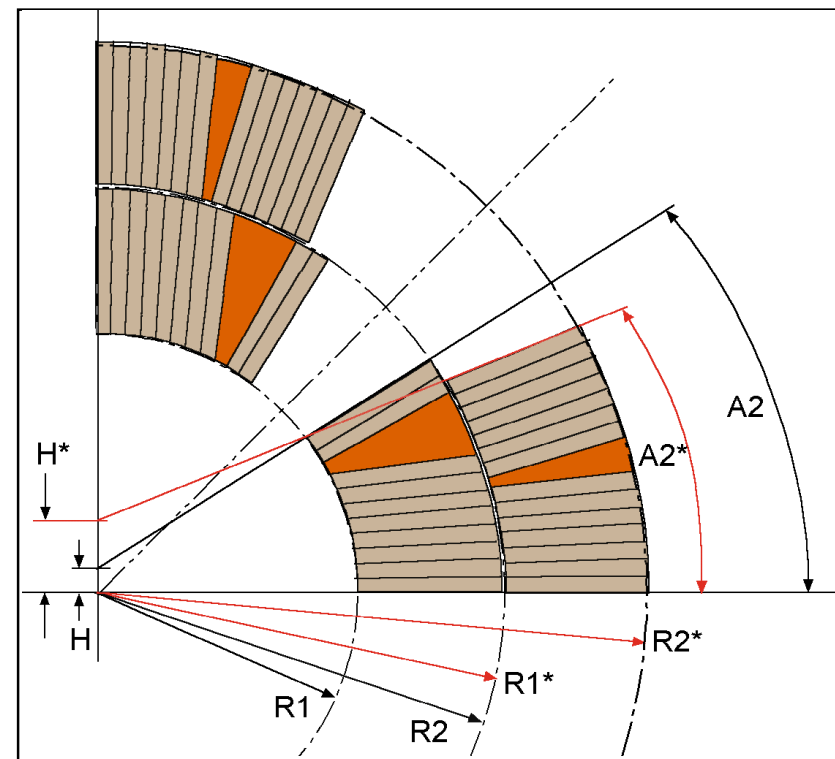

Figure 1. LHC quadrupole coil geometry. (Outer coil parameters are indicated by *)

\begin{tabular}{|c|c|c|c|c|}
\hline Parameter & Inner coil & Outer coil & $\begin{array}{l}\text { Idealized } \\
\text { inner coil }\end{array}$ & $\begin{array}{l}\text { Idealized } \\
\text { outer coil }\end{array}$ \\
\hline A1 & $0^{\circ}$ & $0^{\circ}$ & $0^{\circ}$ & $0^{\circ}$ \\
\hline $\mathrm{A} 2$ & $31.1^{\circ}$ & $22.42^{\circ}$ & $36^{\circ}$ & $30.4^{\circ}$ \\
\hline $\mathrm{H}, \mathrm{mm}$ & 2.53 & 6.65 & 0 & 0 \\
\hline A3 & \multirow{2}{*}{$\begin{array}{c}\text { Tilted } \\
\text { wedge }\end{array}$} & \multirow{2}{*}{$\begin{array}{c}\text { Tilted } \\
\text { wedge }\end{array}$} & $23.4^{\circ}$ & $14.6^{\circ}$ \\
\hline A4 & & & $29.7^{\circ}$ & $17^{\circ}$ \\
\hline $\mathrm{R} 1, \mathrm{~mm}$ & 28.00 & 43.88 & 28 & 43.88 \\
\hline $\mathrm{R} 2, \mathrm{~mm}$ & 43.15 & 59.00 & 43.15 & 59 \\
\hline $\mathrm{R}_{\text {yoke }}, \mathrm{mm}$ & \multicolumn{2}{|c|}{$90, \mu=\infty$} & \multicolumn{2}{|c|}{$90, \mu=\infty$} \\
\hline $\mathrm{R}_{\text {ref, }} \mathrm{mm}$ & \multicolumn{2}{|c|}{10} & \multicolumn{2}{|c|}{10} \\
\hline $\mathrm{A}_{\text {ref }}$ & \multicolumn{2}{|c|}{$0^{\circ}$} & \multicolumn{2}{|c|}{$0^{\circ}$} \\
\hline $\mathrm{J}, \mathrm{A} / \mathrm{mm}^{2}$ & 410 & 410 & 425 & 437 \\
\hline
\end{tabular}


The fields produced by the inner and outer coils are calculated separately and then the harmonic contributions are summed to give the total harmonic for the magnet. Table 2 shows these results and the values from Saclay calculations [4] using an iron yoke with $\mu=\infty$. (Note that the negative sign for the field components is an arbitrary sign convention.) Since the current blocks have been made radial, the allowed multipoles appear higher that those obtained by calculations that consider the tilt of the current blocks.

Table 2. Results for LHC quad field calculation.

\begin{tabular}{|l|r|r|r|r|}
\hline & Inner coil & Outer coil & Total & \multicolumn{1}{l|}{ Saclay value } \\
\hline $\mathrm{B} 1, \mathrm{~T}$ & -1.231 & -0.9149 & -2.15 & 2.23 \\
\hline $\mathrm{B} 5 / \mathrm{B} 1 \times 10^{4}$ & -6.50 & 3.67 & -2.83 & 0.47 \\
\hline $\mathrm{B} 9 / \mathrm{B} 1 \times 10^{4}$ & -2.30 & 2.71 & 0.41 & 0.05 \\
\hline Gradient, T/m & & & -215 & 223 \\
\hline Fx (octant ), $\mathrm{kN} / \mathrm{m}^{*}$ & & & 496 & 540 \\
\hline Fy (octant), kN/m* & & & 780 & 730 \\
\hline
\end{tabular}

* For multilayer examples, forces can be approximated, but not rigorously computed, by superposition. In this case the force on each coil is calculated individually at the field produced by the single layer. Then this force is scaled linearly for each layer by the ratio of the total field produced by both coils to the field produced by the individual coil. The forces for the two layers are then summed to give the total force.

The operating margin can be computed from the ratio of the current density in the superconductor to its critical current density at operating temperature and the maximum field on the conductor, Bmax.

The IMC output includes color-coded graphs of the field strength in the conductor region. (These neglect the field enhancement in the coil ends.) These show that the maximum field on the conductor occurs at the pole turn of the inner coil at its inner radius. However, for a multilayer coil, Bmax must be computed from the contributions of all coil layers. Figure 2 shows the computation of the $\mathrm{x}$ and $\mathrm{y}$ field components due to the outer coil at the inner coil location; note that the input screen has Rref $=28$ and Aref $=36^{\circ}$. The $\mathrm{x}$ and $\mathrm{y}$ field components due to the inner coil must also be computed at this same reference point. For the inner coil, $\mathrm{Bx}=-1.954$ and $\mathrm{By}=-2.822$; for the outer

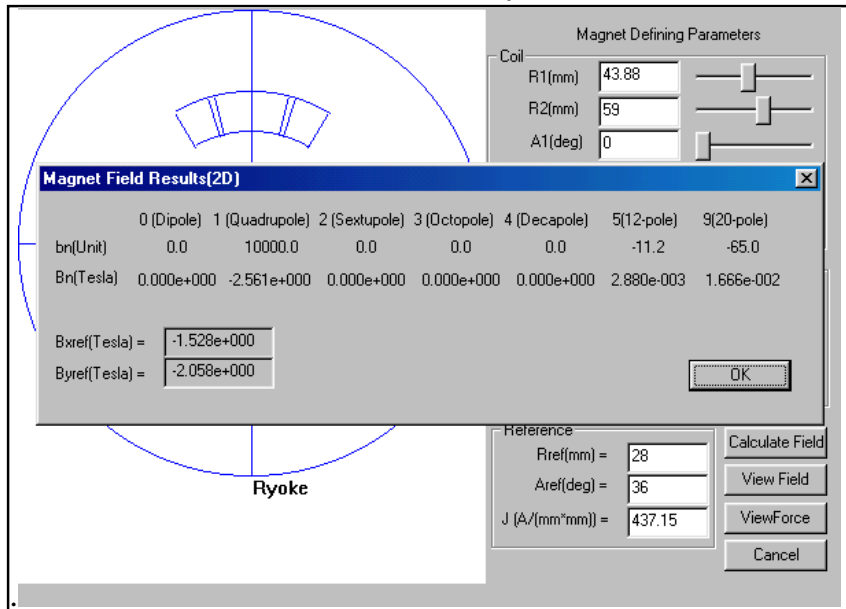

Figure 2. Field component calculation for LHC quad at location of peak field. coil, $\mathrm{Bx}=-1.529$ and $\mathrm{By}=-2.058$. The magnitude of $\mathrm{Bmax}$ is thus 5.995 .

The cable and operating parameters are shown in Table 3. $\mathrm{C}_{0}$ is the coefficient used in the Summer's formula [5] for computing the critical surface of $\mathrm{Nb}_{3} \mathrm{Sn}$.; the value of $\mathrm{C}_{0}$ listed is that set by the VAMAS (Versailles Agreement Meeting on Applied Science) and is used in the magnet tutorial to compute $\mathrm{J}_{\mathrm{C}}$ for $\mathrm{Nb}_{3} \mathrm{Sn}$. For comparison, the Saclay calculation indicated an operating margin of $19.7 \%$ with a peak field on the conductor of $6.85 \mathrm{~T}$.

Table 3. LHC quad operating margin calculation

\begin{tabular}{|l|r|}
\hline $\mathrm{Nb} 3 \mathrm{Sn}$ Strand diameter & 0.78 \\
\hline $\mathrm{Cu} / \mathrm{non}-\mathrm{Cu}$ & 1.7 \\
\hline Strands/cable & 36 \\
\hline Operating current & 11870 \\
\hline Operating temperature & 1.9 \\
\hline $\mathrm{C}_{0}$ & 12,000 \\
$\mathrm{~K}$
\end{tabular}

\begin{tabular}{|l|c|}
\hline Area of cable conductor & $17.20 \mathrm{~mm}^{2}$ \\
\hline $\mathrm{SC}$ area in cable & $6.37 \mathrm{~mm}^{2}$ \\
\hline Current density in conductor & $1863.09 \mathrm{~A} / \mathrm{mm}^{2}$ \\
\hline Peak field on conductor, $\mathrm{B}_{\max }$ & $6.00 \mathrm{~T}$ \\
\hline $\mathrm{J}_{\mathrm{c}} @ \mathrm{~B}_{\max }$ and $\mathrm{T}_{\mathrm{op}}{ }^{*}$ & $2796 \mathrm{~A} / \mathrm{mm}^{2}$ \\
\hline Margin & $33.37 \%$ \\
\hline
\end{tabular}

* $\mathrm{J}_{\mathrm{c}} @ \mathrm{~B}_{\max }$ and $\mathrm{T}_{\mathrm{op}}$ is obtained from the Material Properties Library in the Superconducting Magnet Tutorial. This interactive procedure computes the critical current density as a function of applied field and temperature using the Summer's formula.

\section{U. TWENTE DIPOLE EXAMPLE}

A two-layer dipole design developed by the University of Twente [6], and designated MSUT, was proposed as an LHC dipole prototype. This magnet used powder-in-tube $\mathrm{Nb}_{3} \mathrm{Sn}$ conductor. The cross section of the coils is shown in Figure 3. In this case there are two wedges in the inner coil and both coils have tilted current blocks.

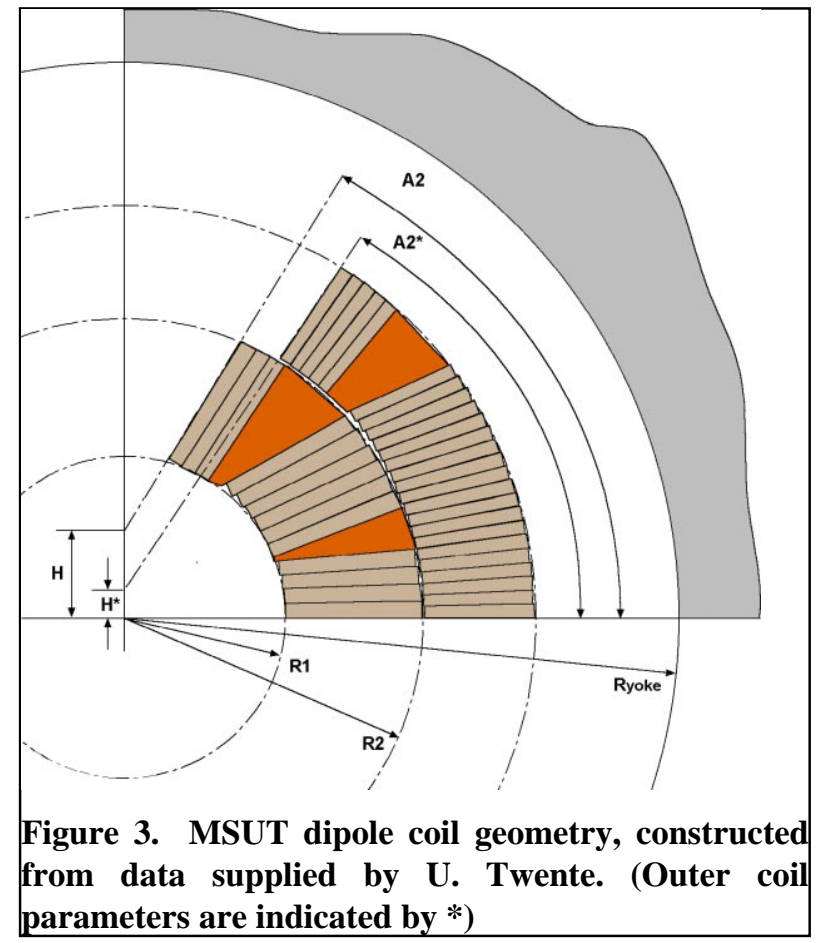


To use the interactive magnetic field calculator (IMC), the inner coil needs to be broken into two separate coils in order to conform to the single wedge limitation. Thus, three separate calculations need to be performed and summed in order to compute the total field in this magnet.

The values of the IMC input parameters for the three idealized coil sections with radial current blocks are listed in Table 4; the angles were determined by visual adjustment to achieve an average position of the radial line. The current density in each coil section was computed using the same method as that described for the LHC quadrupole example.

Table 4. Input parameters for MSUT magnet model using three coil sections.

\begin{tabular}{|l|r|r|r|}
\hline Parameter & Inner coil-1 & Inner coil-2 & Outer coil \\
\hline A1 & $0^{\circ}$ & $21.95^{\circ}$ & $0^{\circ}$ \\
\hline A2 & $18.8^{\circ}$ & $74.56^{\circ}$ & $58.01^{\circ}$ \\
\hline A3 & & $52.32^{\circ}$ & $42.07^{\circ}$ \\
\hline A4 & & $56.68^{\circ}$ & $48.11^{\circ}$ \\
\hline R1, mm & 24.95 & 24.95 & 47.16 \\
\hline R2, mm & 46.75 & 46.75 & 64.56 \\
\hline Ryoke, mm & 105 & 105 & 105 \\
\hline Rref, mm & 12.5 & 12.5 & 12.5 \\
\hline Aref & $0^{\circ}$ & $0^{\circ}$ & $0^{\circ}$ \\
\hline Operating current, A & \multicolumn{3}{|c|}{19100} \\
\hline $\mathrm{J}, \mathrm{A} / \mathrm{mm}^{2}$ & 297.93 & 294.24 & 519.95 \\
\hline
\end{tabular}

The IMC results for the field and Lorentz forces and a comparison with those obtained from U. Twente are shown in Table 5 for the case of iron with infinite or high $\mu$; the B0 results of the calculation for the case of no iron are shown also. These IMC calculations show reasonably good agreement with the U. Twente results, except for the sextupole; as usual, the sextupole from the IMC is much higher than values calculated from the actual design with tilted current blocks. The actual field attained in this magnet should be lower that the values shown because of iron saturation effects; during tests, this magnet attained a field of $11.3 \mathrm{~T}$ at $19.1 \mathrm{kA}$.

Table 5. MSUT Magnet Calculation for Iron without saturation.

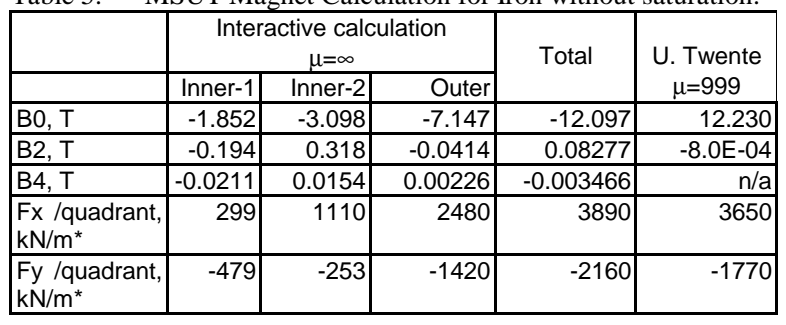

MSUT Magnet Calculation without Iron.

\begin{tabular}{|c|c|c|c|}
\hline & Interactive calculation $\mu=1$ & Total & U. Twente \\
\hline B0, & \begin{tabular}{l|l}
$-2.80 \mathrm{E}+00$ & $-5.60 \mathrm{E}+00$ \\
\end{tabular} & -10.29 & 10.13 \\
\hline
\end{tabular}

The maximum field on the conductor (Bmax) occurs at the inner radius of the pole turn of the inner coil (neglecting the field enhancement in the coil ends). The IMC can be used to compute the field components at that location due to each of the three coil segments, and the results summed. For the case of $\mu=\infty, B x=0.122$ $(=-0.197+0.438-0.119)$ and $\mathrm{By}=-12.584(=-1.338-$ $4.258-6.988$ ); thus $B \max =12.585$. Since iron saturation takes place in this magnet, the peak field on the conductor is not as high as this. We cannot compute a reasonable estimate of the operating margin for this magnet without a precise value of Bmax. Also, the powder-in-tube conductor for this magnet has a higher Jc than that represented by the Summer's formula with $\mathrm{C}_{0}=12,000$.

\section{CONCLUSION}

We have illustrated how the interactive magnetic field calculator (IMC) which is included in the Superconducting Accelerator Magnets CD-ROM tutorial can be used to estimate the magnetic fields and Lorentz forces in multiple layer magnets with more than two wedges per coil.

Two examples of magnets with $\mathrm{Nb} 3 \mathrm{Sn}$ conductor were considered. In both cases, the central field values calculated by the IMC were in close agreement with the designers' calculation results. However, these magnets used tilted current blocks, and thus the IMC approximation to the allowed multipole fields was not very close to the designers' calculation results. For the LHC quadrupole, the operating margin could also be estimated by using the tutorial's interactive calculation of Jc from the Summer's formula.

Although this simple IMC procedure does not give precise results for field harmonics, it can be very helpful in making preliminary comparisons of cosine theta magnet designs.

\section{REFERENCES}

[1] "Superconducting Accelerator Magnets" CD-ROM tutorial
information at www.mjb-plus.com

[2] Meinke, R. B, Goodzeit, C. L. and Ball, M. J., Interactive Procedure for Estimating Performance of Magnet Designs, Poster Paper LFB-06 Presented at the 1998 Applied Superconductivity Conference, September 13-18, Palm Desert CA USA. A PDF copy is available at the web site given in the previous reference.

[3] Dimensional data from LHC Project Magnet Parameters, CERN 01/28/99 and communicated by Arnaud Devred, CEA Saclay, 01/08/97.

[4] Communicated by Arnaud Devred, CEA Saclay, calculation of 02/08/97.

[5] L.T. Summers, J.C. McKinnell, S.L. Bray and J.W. Ekin, "Characterization of Multifilamentary $\mathrm{Nb}_{3} \mathrm{Sn}$ Superconducting Wires for Use in the 45-T Hybrid Magnet", IEEE Trans. Appl.

Superconductivity, vol. 5, no. 2, 1764, June 1995

[6] Data for this magnet was communicated by Andries den Ouden, University of Twente, Faculty of Applied Physics, Feb. 1999. 